Research Article

\title{
Melatonin stimulates extracellular matrix formation in human articular cartilage chondrocytes
}

\author{
Jesús Pintor*1,2 \\ ${ }^{1}$ Pharmacological Research Laboratory, Royal National Academy of Pharmacy, C/ de la \\ Farmacia 11, 28004 Madrid, Spain \\ ${ }^{2}$ Dep. Biochemistry, Faculty of Optometry, Universidad Complutense de Madrid, 28037 \\ Madrid, Spain \\ *Correspondence: E-Mail: Jpintor@ucm.es; Tel +34913946859
}

Running title: Melatonin stimulates human chondrocytes.

Received January 28, 2019; Accepted February 10, 2019

\begin{abstract}
The articular cartilage present in joints is permanently subjected to physical stress, thus producing injuries and consequently pain. Chondrocytes are responsible for repairing this damage, but often the reparation tissue is inadequate. The application of melatonin to human articular chondrocytes may be a better solution, by generating a good extracellular matrix. Human chondrocytes were assayed in the presence of melatonin, demonstrating that these cells generate more extracellular matrix indicated by the increased Alcian blue staining. This effect of melatonin was concentrationdependent and presented a $\mathrm{pD}_{2}$ value of $6.8 \pm 0.1(\mathrm{n}=5)$. Melatonin also significantly promotes the production of collagen II quantified by western-blot analysis. The effect of melatonin on the collagen II production is also concentration-dependence, with a $\mathrm{pD}_{2}$ value of $6.9 \pm 0.2(n=5)$. In contrast, melatonin had little effect on the collagen $X$. The antagonistic study by its non-selective membrane receptor blocker luzindole or its $\mathrm{MT}_{2}$ specific blocker DH-97 identified that these actions of melatonin are mediated by melatonin membrane receptors, mainly by $\mathrm{MT}_{2}$. In summary, melatonin stimulates the production of extracellular matrix and collagen II in chondrocytes and these actions may have significantly clinical implications since it may benefit the autologous chondrocyte transplantations, the only way to maintain and repair the damaged cartilage.
\end{abstract}

Keywords: melatonin, cartilage, chondrocytes, extracellular matrix, melatonin membrane receptor.

\section{INTRODUCTION}

The articular cartilage, which is located in the synovial joints of relevant bones, such as knees or ankles, is the most common type of cartilage, characterized by being rich in proteoglycans as well as collagen II. The cartilage is continuously subjected to great stress, and, thus it demands the substantial replacement with the extracellular matrix which is produced by the chondrocytes (1). An excess of cartilage extracellular matrix 
loss will produce pathologies that can incapacitate the individual from living a normal life (2). The only way to restore the damaged cartilage is by surgery, which is often combined with autologous chondrocyte transplantation. Basically, a sample of healthy cartilage is taken and cells are multiplied, these cells are then implanted in the damaged cartilage after removing the injured area (3).

Several strategies have been carried out to perform chondrocyte implantation, nonetheless, no studies have been tried to stimulate these cells to produce more extracellular matrix. Physiologically, if more extracellular matrix was generated, after the chondrocyte implantation, the healing and repair processes of the cartilage would be accelerated.

An interesting molecule with a variety of physiological actions is melatonin. Melatonin, either acting by itself, or by stimulating melatonin membrane receptors, mediates many relevant activities such as the control of free radicals $(4,5)$. It can stimulate lymphocyte maturation (6) or regulates some physiological processes among many other actions $(7,8)$.

In the cartilage, the activities of melatonin have been observed in normal and pathological processes such as osteoarthritis (9) or intervertebral disc degeneration, where melatonin acts via Sirt1 expression (10). Moreover, melatonin can ameliorate rheumatoid arthritis reducing inflammation, limiting cartilage degradation and bone erosion (11).

The articular cartilage is lack of vascularization, thus this feature limits the circulatory melatonin reaching the area and therefore this may contribute to chondrocyte malfunction. Nonetheless, melatonin has been described in the human synovial fluid (12) and as such it may plays a critical role in the physiology of chondrocytes. Till now, the exact function(s) of the synovial melatonin is not clear. We speculate that melatonin may stimulate the production of extracellular matrix in the human cartilage. This possibility has never been fully investigated yet. To test this hypothesis, in the present study, the potentially stimulatory effects of melatonin on the production of extracellular matrix is explored. In addition, collagen II, one of the most relevant proteins presented in extracellular medium, is also measured when human chondrocytes are treated with melatonin.

\section{MATERIALS AND METHODS}

\subsection{Human articular chondrocytes}

Human chondrocytes, used throughout the study, were obtained from the articular cartilage (knees) of healthy individuals $(n=5)$. These cells were kindly provided by Dr. Pedro Guillén (Clinica CEMTRO, Madrid, Spain), and followed the Ethical Committee Standards of Clinica CEMTRO.

Each piece of healthy cartilage was placed in a petri dish and cut into small portions using a sterile razor blade. The fragments were carefully placed in a sterile $50 \mathrm{~mL}$ tube and isolation of cells was performed by enzymatic treatment with $1 \mathrm{mg} / \mathrm{mL}$ collagenase A (Roche Diagnostics GmbH, Mannheim, Germany) at $37^{\circ} \mathrm{C}$ overnight. The processed samples were filtered through a $70 \mu \mathrm{m}$ gauge nylon cell strainer (BD Falcon, Franklin Lakes, NJ) connected to a clean $50 \mathrm{~mL}$ tube. To recover the maximum number of cells, the cell strainer was washed with $10 \mathrm{~mL}$ of DMEM. The sample was centrifuged for 5 minutes at $1,800 \mathrm{rpm}$ at room temperature discarding the supernatant. After adding other $20 \mathrm{ml}$ of DMEM at room temperature, the supernatant was discarded, and additional $20 \mathrm{~mL}$ of DMEM was added to the tube to be further centrifuged under the 
same conditions. The pellet was re-suspended and cultured in high glucose Dulbecco's modified Eagle's medium (Gibco/Invitrogen, Carlsbad, CA) containing 10\% foetal bovine serum (Sigma-Aldrich, St. Louis, MO) and 0.05 penicillin/ streptomycin (Gibco/Invitrogen) at $37^{\circ} \mathrm{C}$ in humidified atmosphere with $5 \% \mathrm{CO}_{2}$ and $95 \%$ air.

\subsection{Extracellular matrix analysis}

Cartilage matrix deposition in human articular chondrocytes was measured using Alcian blue staining. Cells were seeded at a density of $2 \times 10^{4}$ cells/well in six-multiwell dishes. When cells reached the confluence, on the 3rd day, cells were treated with melatonin at the given concentration. The cells were cultured with fresh medium containing melatonin (or/and any other given compound) every 3 days. Cultures were monitored over a total period of 15 days. At different time points, 3, 7, 10 and 15 days, extracellular matrix was analysed using Alcian Blue staining as previously described (13).

\subsection{Cell viability}

Cell viability throughout the whole experimental period (15 days) was performed by using trypan blue staining. Viable (unstained) cells were counted using a hemocytometer under the light microscope. Experiments were performed in triplicate.

\subsection{Melatonin concentration-response studies}

The concentration-response curves, either measuring extracellular matrix deposition or protein quantification by western-blot, were performed by treating the cells with different concentrations of melatonin ranging from $10^{-9}$ to $10^{-4} \mathrm{M}$, and the extracellular matrix or protein levels were measured at the $15^{\text {th }}$ day of the culture. The medium containing the given concentration of melatonin was changed every three days.

\subsection{Western-blot analysis}

To analyze and quantify both, collagen II and collagen X, lysates from each well (45 $\mu \mathrm{g}$ of protein) were subjected to $10 \%$ SDS-polyacrilamide gel electrophoresis to be further transferred to nitrocellulose membranes. Afterwards, the membranes were blocked and incubated overnight with collagen II and collagen X antibodies (ab188570 and ab58632, respectively, abcam, Cambridge, USA) at a dilution of $(1: 1,000)$. After washing, blots were incubated with peroxidase-conjugated secondary antibody. Development was performed using ECL system (Amersham, Buckinghamshire, UK).

In order to verify the correct loading of the samples, membranes were stripped in $62.5 \mathrm{mM}$ Tris- $\mathrm{HCl} \mathrm{pH} \mathrm{6.8,2 \%} \mathrm{SDS} \mathrm{and} 100 \mathrm{mM} \mathrm{2-mercaptoethanol} \mathrm{and} \mathrm{re-blotted}$ using ERK-2 antibody. Films were scanned and densitometric analysis was carried out using Kodak GL 200 Imaging system and Kodak Molecular Imaging software (Kodak, Rochester, NY, USA). All data shown are results representative of five independent experiments.

\subsection{Melatonin membrane receptor antagonistic studies}

To demonstrate whether melatonin membrane receptors are involved in this action, two melatonin membrane receptor antagonists were used, that are, the non-selective 
melatonin receptor antagonist luzindole and the $\mathrm{MT}_{2}$ selective antagonist $\mathrm{DH}-97$ (Tocris, Bristol, UK). Each antagonist alone, or both together, were pre-incubated for 30 min at a concentration of $100 \mu \mathrm{M}$ before the melatonin $(100 \mu \mathrm{M})$ was applied. Fresh medium containing the given concentration of the antagonists and melatonin was changed every three days.

\subsection{Statistical analysis}

All the plots and the statistical analysis were carried out with Prism Graph Pad 7 (GraphPad Software, CA, USA). Student's t-test statistical analysis was carried out when necessary. The statistical significance is set up $p<0.05$ and has been indicated in each case.

\section{RESULTS}

\subsection{Effect of melatonin on extracellular matrix production in chondrocytes}

Extracellular matrix production was evaluated by Alcian blue staining in human articular chondrocytes, treated either with vehicle or with a single dose of melatonin $(100 \mu \mathrm{M})$. The results showed that melatonin treatment significantly increased the extracellular matrix of chondrocytes at the day 15 th of treatment $(n=5)$ indicted by the strong Alcian blue staining. In the control group, Alcian blue staining was significantly weaker than that in the melatonin treated group (Fig. 1A).
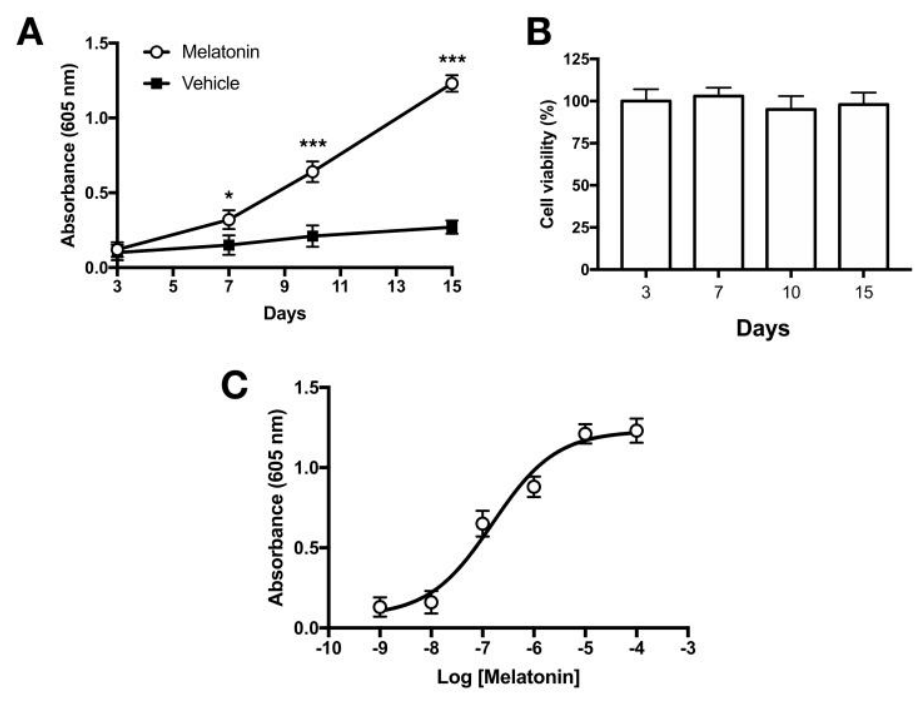

Figure 1. Effect of melatonin on extracellular matrix production in articular chondrocytes.

A: Chondrocytes were treated with a single dose of melatonin $(100 \mu \mathrm{M})$. At the indicated times, extracellular matrix was quantified by Alcian Blue staining of cell layers, following solubilisation. Dye uptake was quantified at $605 \mathrm{~nm}\left({ }^{*} p<0.05\right.$, $* * * p<0.001$, vs. vehicle; $n=5)$. B: Viable chondrocytes were determined by trypan blue exclusion assay. The number of viable cells in the control (without treatment) was normalized to $100 \%$ and was used as the reference for cells treated with melatonin. $C$ : Concentration-response curve of melatonin and extracellular matrix (measuring in the absorbance at $605 \mathrm{~nm}$ ) in the presence of concentrations of melatonin from $10^{-9} \mathrm{M}$ to $10^{-4} M(n=5)$. 
As to the cell viability throughout the 15 days of the study, there were no statistically differences to be observed between the groups $(n=5)$ determined by trypan blue staining (Fig. 1B).

A concentration-response study showed that the effect of melatonin to stimulate the extracellular matrix formation of chondrocytes is dose dependent and the pD2 value was $6.8 \pm 0.1(\mathrm{EC} 50=0.15 \mu \mathrm{M})(\mathrm{n}=5)($ Fig. $1 \mathrm{C})$.

\subsection{Effect of melatonin on collagen II and collagen $\mathrm{X}$ production}

After the observation of melatonin promoting the production of extracellular matrix in chondrocytes, the levels of two relevant collagens (collagen II and collagen X) both contributing to the extracellular matrix, were assayed at the day 15 th of the study. In this sense, the concentration-response curves of melatonin (ranging from $10^{-9}$ to $10^{-4} \mathrm{M}$ ) on collagen II and collagen $X$ were performed. The results showed that the concentration-dependent effects of melatonin on collagen II formation was obvious and the maximal effective concentration was around $10^{-5} \mathrm{M}(\mathrm{n}=5)$. On the contrary, melatonin had little effect on collagen $\mathrm{X}$ formation (Fig. 2).
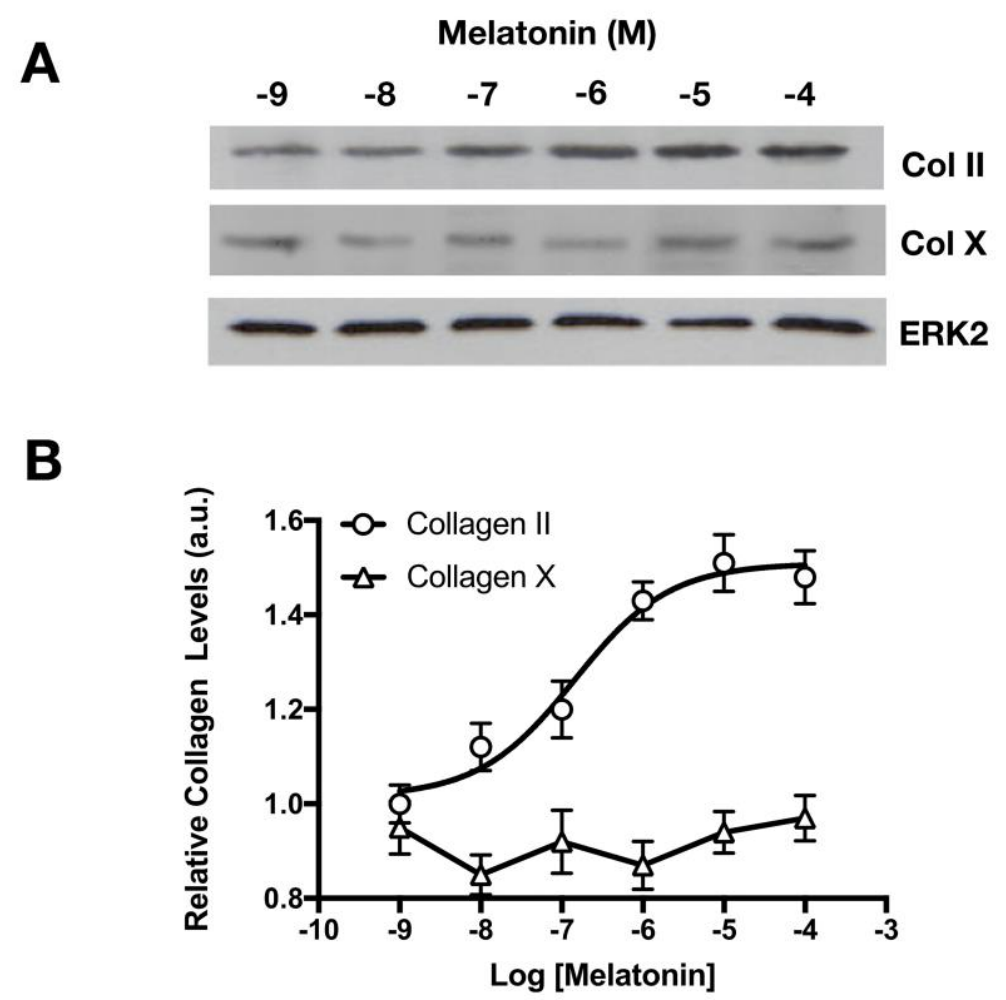

Figure 2. The concertation dependent studies of melatonin on collagen II and $X$ expression.

A: The protein expression of collagen II was concentration dependently upregulated by melatonin (panel $A$, upper gel) while melatonin failed to upregulate the expression of collagen X (panel A, middle gel)._B: Plot of the results obtained in panel A to obtain the corresponding concentration response curves for collagen II and X. Data (mean $\pm S D$ ) $(n=5$ are represented in arbitrary units (a.u.) and normalized to the intensity of the band corresponding control (ERK2). 
The calculated $\mathrm{pD}_{2}$ value for collagen II is $6.9 \pm 0.2\left(\mathrm{EC}_{50}=0.12 \mu \mathrm{M}\right)(\mathrm{n}=5)$. Since there were no changes in collagen $\mathrm{X}$ levels, it was not possible to calculate a concentration-response curve $(n=5)$.

3.3 Effect of melatonin membrane receptor antagonists on the collagen II formation at the presence of melatonin.

The effect of melatonin on collagen II production was studied in the presence of the melatonin membrane receptor antagonists luzindole and DH-97. The non-selective membrane receptor antagonist luzindole, at $100 \mu \mathrm{M}$, partially blocked melatonin (100 $\mu \mathrm{M})$ effects on collagen II formation with a reduction of $33.3 \pm 3.6 \%(n=5)$. When the selective $\mathrm{MT}_{2}$ antagonist $\mathrm{DH}-97$ was used, this reduction on collagen II formation was increased to $73.3 \pm 2.4 \%(n=5)$. When both antagonists were tested together the effect of melatonin on collagen II formation was further significantly abolished $(n=5)$.

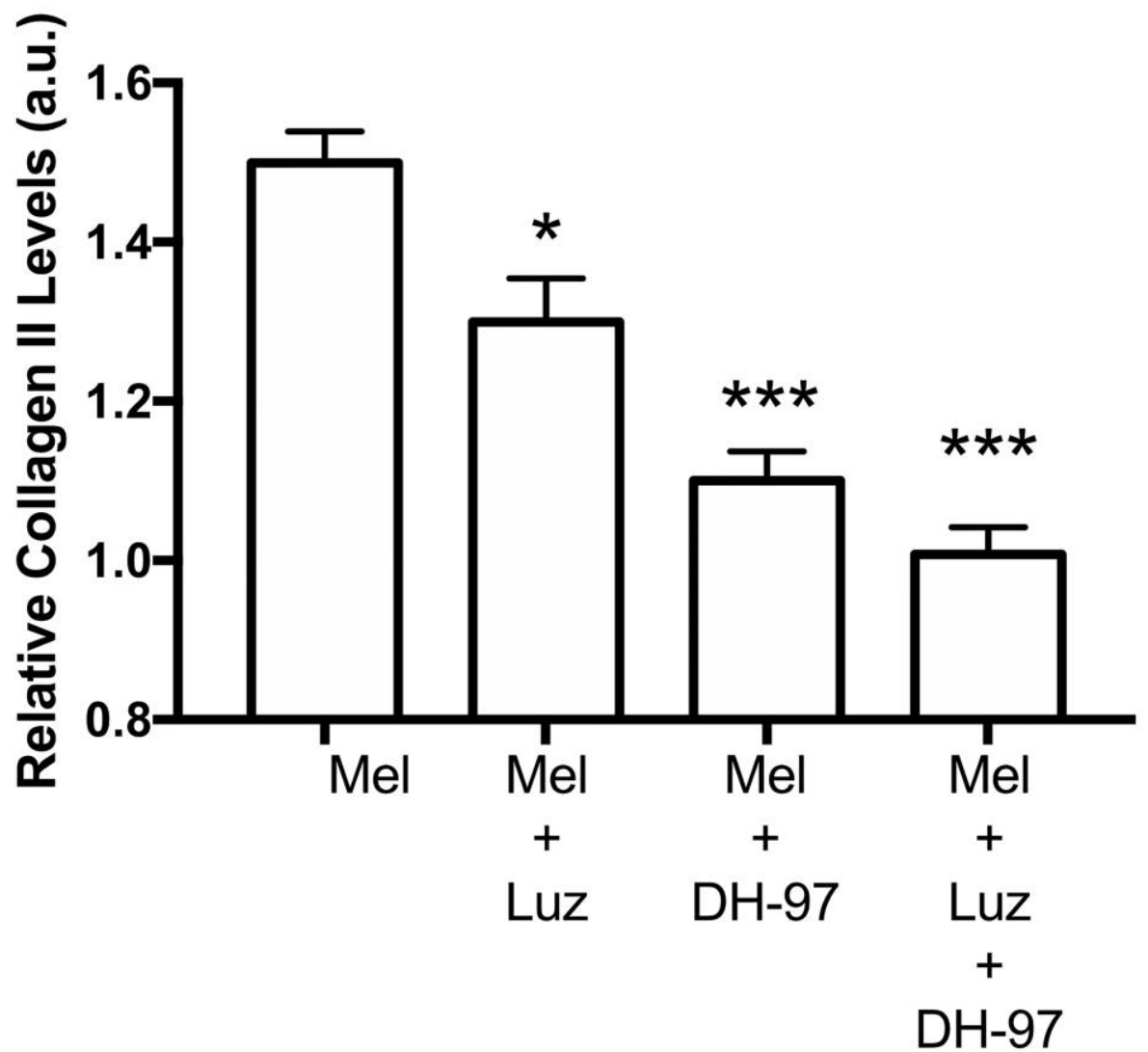

Figure 3.- Effect of melatonin membrane receptor antagonists on collagen II formation induced by melatonin.

The effect of melatonin $(100 \mu M)$ on the expression of collagen II was partially antagonized by luzindole $(100 \mu M)$ and a stronger antagonization was observed by $D H$ $97(100 \mu M)$. The combination of both antagonists provided further inhibition on collagen II production at the present of melatonin $\left({ }^{*} p<0.05, * * * p<0.001\right.$, vs. vehicle; $n=5$ ). 


\section{DISCUSSION}

It has been observed in the current study that melatonin stimulates the physiological function of human articular chondrocytes to increase the production of extracellular matrix. Further study indicated that one of the most representative components of extracellular medium, collagen II, was also significantly elevated after melatonin treatment. The results presented here are consistent with the previous report, which also showed that melatonin enhanced the production of extracellular matrix in porcine chondrocytes (14).

Although the perspective of that study was to target the treatment of osteoarthritis, it was found that melatonin was able to induce changes in collagen II but was unable to modify collagen X expression. Collagen II in the extracellular matrix is the base where the proteoglycans, which form the cartilage extracellular milieu, carry out their turnover (15). This observation provides a plausible explanation as to why there is not only an increase in collagen II, but also a robust increase in the extracellular medium in the present study.

The lack of changes of collagen $X$ when cells are treated with melatonin are not unusual since this type of collagen is more relevant in endochondral chondrocytes which are responsible of bone growth. In endochondral chondrocytes, collagen $\mathrm{X}$ is a marker for new bone formation and the increase in this protein indicates chondrocyte hypertrophy as a step prior to ossification (16).

Chondrocytes present in the cartilage with a correct turnover of the extracellular matrix are responsible for the perfect performance of joints. Excessive sports training, or simply aging, can lead to cartilage damage (17-19). If the chondrocytes are unable to renew the cartilage in an effective way, patients will suffer pain. Very often, the cartilage generates an abnormal extracellular matrix, termed reparation tissue, which is amorphous and results in pain and joint problems (20). To solve these abnormalities, surgery by means of autologous chondrocyte transplantation with the techniques of ICA or ICAM are currently available. Even though the newly generated extracellular matrix after autologous chondrocyte transplantation is not the same as the native tissue generated it is still better than that the reparation of tissue (3).

In the present work, it has been demonstrated that chondrocytes treated with melatonin improve the ability to produce more extracellular matrix. Melatonin triggers the formation of proteoglycans, but also contributes to the production of a relevant protein of collagen II. The effects of melatonin on either extracellular matrix or collagen II formation are concentration-dependent and its effects can be blocked by melatonin membrane receptor antagonists. Although the pharmacological characterization is preliminary, there is clear evidence that most of the effects in the present work are mediated by $\mathrm{MT}_{2}$. From this point of view, the use of selective agonists of the $\mathrm{MT}_{2}$ receptors such as IIK7, may produce a more robust effect than that observed in melatonin. More experiments are necessary to confirm this hypothesis.

Collectively, melatonin, via its receptors mediated actions, can improve the physiological functions of articular cartilage chondrocytes to increase the extracellular matrix production. This aspect is very clinical relevant, since without an appropriate extracellular matrix production all the damage induced by the physical exercise or even aging, would not be properly healed in joint (21-24). Our results suggest an alternative that chondrocytes used for autologous transplantation can be pre-treated with melatonin to improve the outcome of cartilage repairing clinically. 


\section{ACKNOWLEDGEMENTS}

This work has been supported by The Royal Academy National of Pharmacy and the Grants (Ministerio de Economia y Comptetitvidad) SAF2013-44416R and SAF201677084R.

\section{AUTHORSHIP}

All this work has been designed, the experiments performed, the data analysis, plots and the manuscript elaboration by Dr. Jesus Pintor.

\section{CONFLICT OF INTEREST}

The author declares no conflict of interest.

\section{REFERENCES}

1. Liu C, et al. (2018) Protective effects of the pericellular matrix of chondrocyte on articular cartilage against the development of osteoarthritis. Histol. Histopathol. 33 (8): $757-764$.

2. Tsumaki N (2018) Cartilage/chondrocyte research and osteoarthritis. Regeneration of articular cartilage damage using iPS cell-derived cartilage. Clin. Calcium 28 (6): 803-808.

3. Lopez-Alcorocho JM, et al. (2018) Cartilage defect treatment using high-density autologous chondrocyte implantation: two-year follow-up. Cartilage 9 (4): 363 369.

4. Chang CC, et al. (2018) Protective effect of melatonin against oxidative stressinduced apoptosis and enhanced autophagy in human retinal pigment epithelium cells. Oxid. Med. Cell Long. 2018: 9015765.

5. Manchester LC, et al. (2015) Melatonin: an ancient molecule that makes oxygen metabolically tolerable. J. Pineal Res. 59 (4): 403-419.

6. Calvo JR, Gonzalez-Yanes C, \& Maldonado MD (2013) The role of melatonin in the cells of the innate immunity: a review. J. Pineal Res. 55 (2): 103-120.

7. Alarma-Estrany P \& Pintor J (2007) Melatonin receptors in the eye: location, second messengers and role in ocular physiology. Pharmacol. Therapeutics 113 (3): 507-522.

8. Jockers R, et al. (2016) Update on melatonin receptors: IUPHAR Review 20. Br. J. Pharmacol. 173 (18): 2702-2725.

9. Hosseinzadeh A, et al. (2016) Apoptosis signaling pathways in osteoarthritis and possible protective role of melatonin. J. Pineal Res. 61 (4): 411-425.

10. Zhang Z, et al. (2019) Melatonin protects vertebral endplate chondrocytes against apoptosis and calcification via the Sirt1-autophagy pathway. J. Cell Mol. Med. 23 (1): 177-193.

11. Huang CC, et al. (2019) Melatonin attenuates TNF-alpha and IL-1beta expression in synovial fibroblasts and diminishes cartilage degradation: implications for the treatment of rheumatoid arthritis. J. Pineal Res. e12560: doi: 10.1111/jpi.12560. 
12. Maestroni GJ, Sulli A, Pizzorni C, Villaggio B, \& Cutolo M (2002) Melatonin in rheumatoid arthritis: synovial macrophages show melatonin receptors. Ann. NY. Acad. Sci. USA 966: 271-275.

13. Lunstrum GP, et al. (1999) Chondrocyte differentiation in a rat mesenchymal cell line. J. Histochem. Cytochem. 47 (1): 1-6.

14. Pei M, He F, Wei L, \& Rawson A (2009) Melatonin enhances cartilage matrix synthesis by porcine articular chondrocytes. J. Pineal Res. 46 (2): 181-187.

15. Tiku ML \& Madhan B (2016) Preserving the longevity of long-lived type II collagen and its implication for cartilage therapeutics. Ageing Res. Rev. 28: 62-71.

16. Shen $\mathrm{G}$ (2005) The role of type $X$ collagen in facilitating and regulating endochondral ossification of articular cartilage. Orthodon. Craniofacial Res. 8 (1): 11-17.

17. Hollander AP, et al. (1995) Damage to type II collagen in aging and osteoarthritis starts at the articular surface, originates around chondrocytes, and extends into the cartilage with progressive degeneration. J. Clin. Invest. 96 (6): 2859-2869.

18. McDonough AL (1981) Effects of immobilization and exercise on articular cartilage-a review of literature. J. Orto. Sport Phys. Ther. 3 (1): 2-5.

19. Armstrong SJ, Read RA, Ghosh P, \& Wilson DM (1993) Moderate exercise exacerbates the osteoarthritic lesions produced in cartilage by meniscectomy: a morphological study. Osteoarthr. Cartil. 1 (2): 89-96.

20. Armiento AR, Alini M, \& Stoddart MJ (2018) Articular fibrocartilage - Why does hyaline cartilage fail to repair? Adv. Drug Deliver Rev. doi 10/1016/j.addr.218.12.015.

21. Amis AA (2001) Evaluating the healing of articular cartilage defects. J. Bone Joint Surg. 83A (10):1591-1592.

22. Bae DK, Yoon KH, \& Song SJ (2006) Cartilage healing after microfracture in osteoarthritic knees. Arthroscopy 22 (4): 367-374.

23. Buckwalter JA (1998) Articular cartilage: injuries and potential for healing. $J$. Ortho. Sport Phys. Ther. 28 (4): 192-202.

24. Campbell CJ (1969) The healing of cartilage defects. Clin. Ortho. Rel. Res. 64: 4563.

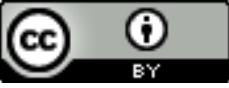

This work is licensed under a Creative Commons Attribution 4.0 International License

Please cite this paper as:

Pintor, J. 2019. Melatonin stimulates extracellular matrix formation in human articular cartilage chondrocytes. Melatonin Research. 2, 1 (Feb. 2019), 106-114. DOI:https://doi.org/https://doi.org/10.32794/mr11250014. 